\title{
Cystoscopic Removal of Migrated Ventriculoperitoneal Shunt Tube: A Case Report with Short Review
}

\author{
T. P. Jeyaselvasenthilkumar ${ }^{1}$ Mohan Sampathkumar $^{1}$ \\ ${ }^{1}$ Department of Neurosurgery, SRM Medical College and Research \\ Centre, Kattankulathur, Chennai, Tamil Nadu, India \\ Indian J Neurosurg 2016;5:120-123.
}

C. Sekar ${ }^{1} \quad$ Mohammad Abith Ali ${ }^{1} \quad$ Y. Premchand ${ }^{1}$

Address for correspondence T. P. Jeyaselvasenthilkumar, MCh (Neuro), F4, Olympia Block, RC Westminster, 46, Kamaraj Road, Kodungaiyur, Chennai, Tamil Nadu 600118, India (e-mail: drjselva@gmail.com).

\begin{abstract}
Keywords

- shunt

- bladder

- perforation

Hydrocephalus is most commonly treated by ventriculoperitoneal (VP) shunt surgery. The distal end of the VP shunt is commonly placed in the peritoneal cavity. Various complications of the distal end of the shunt have been described in the literature. Migration of the distal end of the shunt tube into the bladder is an extremely rare complication of VP shunt surgery. Only three cases of erosive bladder perforation by a peritoneal catheter have been documented so far in the literature. We report an unusual case of perforation of the distal end of the VP shunt into the bladder with encrustation at the tip. Cystoscopic removal of the migrated distal end of the shunt tube is performed. Cystoscopic removal is simple and avoids the complications of cystostomy. The various other complications are shortly reviewed.
\end{abstract}

\section{Introduction}

Ventriculoperitoneal (VP) shunt for diversion of the cerebrospinal fluid (CSF) is the time tested treatment for managing hydrocephalus, though attended by higher rates of complications. ${ }^{1}$ Hollow viscus perforation by the distal end of shunt tube is a rare but well-known complication. The various hollow viscus perforation reported in the literature are stomach, intestine, colon, gall bladder, and uterus. The other common complications reported are infection, dysfunction, CSF cyst, and ascitis. ${ }^{2}$ The incidence of bowel perforation ranges between 0.1 and $0.7 \%{ }^{3}$ Urinary bladder perforation is still rare, only three cases reported so far in the literature. Here, we discuss the complications of VP shunt with special emphasis on urinary bladder perforation and its management with an illustrative case.

\section{Complications of Ventriculoperitoneal Shunt}

\section{Infection}

Shunt infection rate ranges from 1.5 to $38 \%$. Age seems to be an important risk factor, with neonates and young children frequently affected. Shunt infection leads to severe morbidity for the patient. Of even greater concern is the infection-related mortality, with rates up to $20 \%$ reported in the literature. Exteriorization, infection control, and reinsertion of shunt tube is the standard accepted treatment procedure for shunt infection.

\section{Dysfunction}

VP shunt dysfunction ranges between 5 and 30\%. The proximal end of the shunt tube is the one most likely to get blocked. The blockage is usually caused by choroid plexus or blood generated during the catheter placement. The catheter exerts a suction effect which brings choroid plexus, blood, and debris toward the tube, which obstructs the holes in the tube. Distal end occlusions are less common than proximal ones. If the used shunt has a distal slit valve, progressive debris accumulation may lead to its occlusion. Malfunction is also reported if the peritoneum loses its absorptive capacity. This will typically present as increasing abdominal girth. An abdominal ultrasound will show intraperitoneal fluid collection. The malfunctioning shunt tube needs to be revised.
License terms of India received

September 22, 2015 accepted after revision December 18, 2015 published online June 8, 2016
DOI http://dx.doi.org/ 10.1055/s-0036-1581980. ISSN 2277-954X. 


\section{Cerebrospinal Fluid Cyst or Ascitis}

The various etiologies ${ }^{4}$ observed were

1. extracranial metastasis of thalamic glioblastoma through VP shunt tube into the abdomen,

2. VP shunt for hydrocephalus secondary to third ventricular choroid plexus papilloma,

3. shunt infection in post-tuberculous meningitic hydrocephalus, and

4. Craniopharyngioma with hydrocephalus who underwent bilateral VP shunt.

\section{Hollow Viscus Perforation}

Incidence of hollow viscus perforation is less than $1 \%$. The various hollow viscus perforation reported in the literature are stomach, intestine, colon, gall bladder, and uterus in the descending order. The incidence of bowel perforation ranges between 0.1 and $0.7 \%$. Urinary bladder perforation is still rare, only three cases reported so far in the literature. The management of hollow viscus perforation is multidisciplinary, involving various surgical departments. The already reported cases of urinary bladder perforation were managed by cystostomy to remove the perforated shunt tube followed by treatment of infection and reinsertion of shunt tube. We present a novel technique of cystoscopic removal of the migrated shunt tube, which clearly has the advantage of avoiding bladder incision, lesser hospital stay, and lesser morbidity.

\section{Case Report}

A 21-year-old male patient underwent VP shunt for cerebellar astrocytoma with obstructive hydrocephalus followed by tumor excision 10 years ago elsewhere, now admitted with abdominal pain and recurrent urinary tract infection for past 1 month. He did not have headache or vomiting. On examination no focal neurological deficit, no signs of meningeal irritation, no evidence of raised intracranial pressure, no tenderness along the shunt tube tract, shunt pump collapsed, and no filling observed. Ultrasound of the kidneys, ureters, and bladder revealed VP shunt tube's distal end perforating into the urinary bladder and tip seen within the lumen with encrustation of the tip. Computed tomography (CT) of the abdomen confirmed the findings of ultrasound and ruled out free fluid in the peritoneal cavity. CT brain showed the shunt tube tip in brain parenchyma and no hydrocephalus. After doing preliminary investigations, patient planned for shunt tube removal by the multidisciplinary approach (neurosurgery and urology department). Under endotracheal tube general anesthesia, cystoscope was done and shunt tube tip visualized intraluminally (-Figs. 1 and 2 ) with crust formation in the tip, which extends proximally for approximately $2 \mathrm{~cm}$ (-Figs. 3 and 4). The abdominal incision was made in the previous scar and a shunt tube exposed. Fibrous tissue around the shunt tube anchoring it to the peritoneum was released. The intraluminal portion of the shunt tube seen through cystoscope was cut with scissors through the cystoscope at the proximal most visible portion and removed per urethra through the cystoscope. The encrustation at the tip

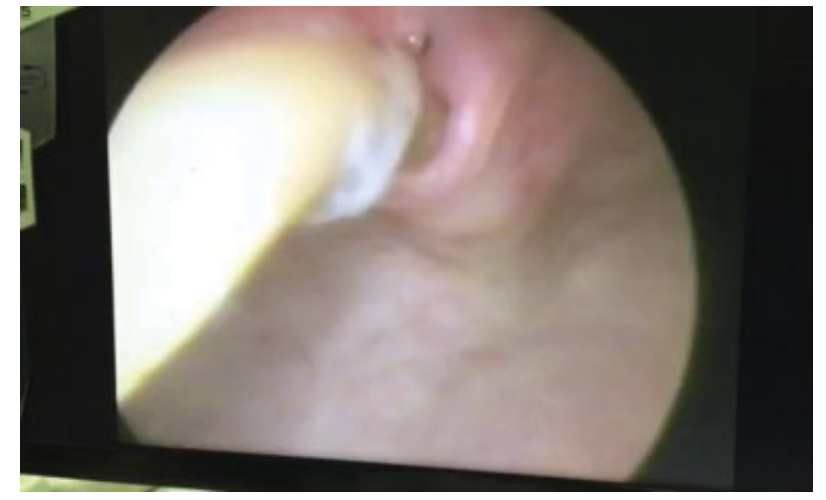

Fig. 1 Cystoscopic view of perforated shunt tube.

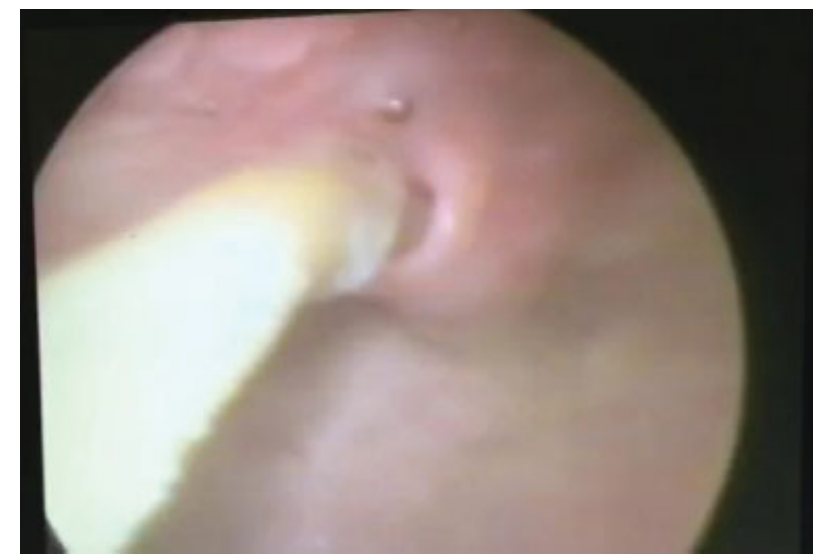

Fig. 2 Adhesion between shunt tube and bladder mucosa released.

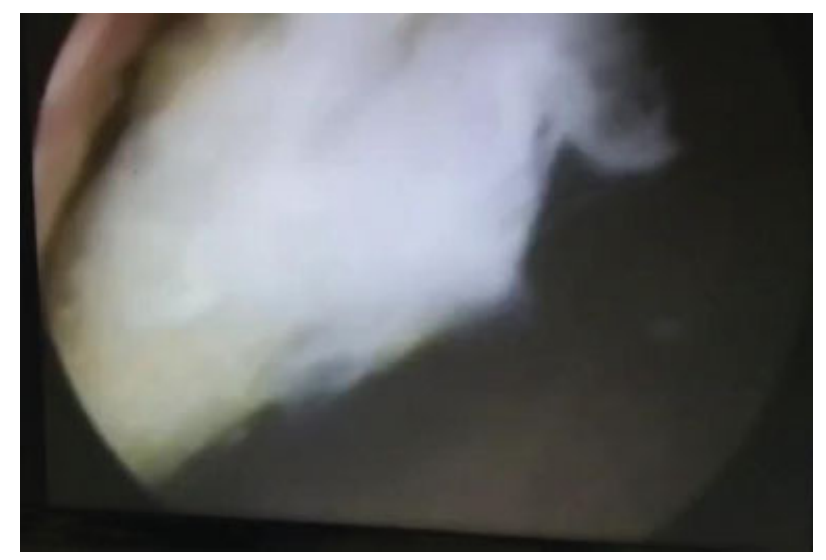

Fig. 3 Encrustation at the tip.

was thin and can be removed per urethra without damaging the same. The proximal end was removed through cranial incision. Postoperatively, the patient was catheterized and the catheter was removed after 3 weeks. Since patient was shunt independent, no further VP shunt was planned. The patient is doing well.

\section{Discussion}

The incidence of perforation of the distal end of the VP shunt into the bladder is very rare. The location of the bladder 


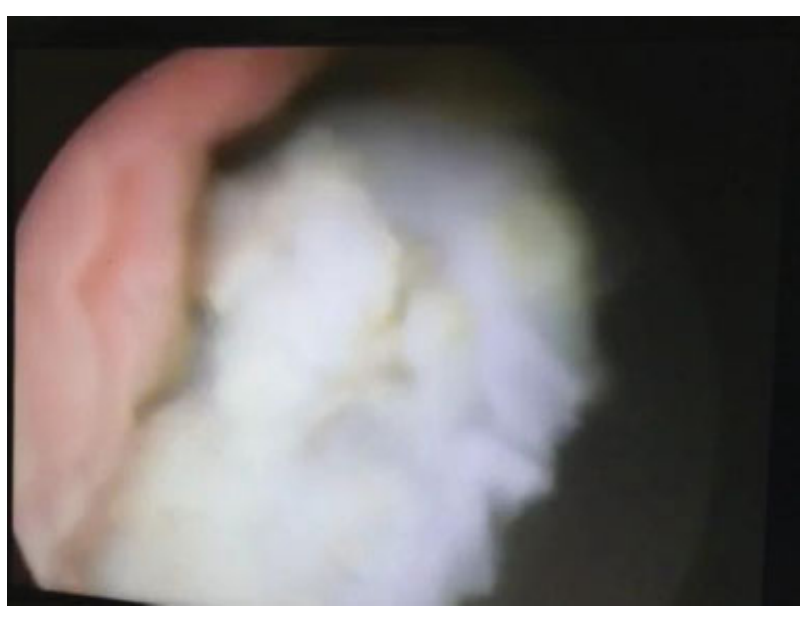

Fig. 4 Magnified view of encrustation.

makes it a highly unlikely site for peritoneal catheter penetration, as the catheter should reach the extraperitoneal space to perforate the bladder. There are reports in the literature stating the extrusion of peritoneal catheter of the VP shunt through the ureter, case of bladder calculus formation and urinary retention secondary to the perforation of a normal bladder by a VP shunt ${ }^{5}$. In our case, the patient presented with lower urinary tract symptoms. A high index of suspicion is required when making the diagnosis. Diagnosis can be established by ultrasonogram and CT scan of the abdomen and the pelvis, which identifies the distal end of the shunt tube in the bladder. A cystourethroscopy has to be done to confirm the diagnosis. The exact cause of this perforation is not known. The postulated mechanism states that the freely moving peritoneal catheter becomes adherent to a hollow viscus and gets anchored to the serosa. Presence of continuous peristaltic movements of the gut coupled with the water hammer effect of the CSF pulsations along with beveled end of the shunt tube penetrates the wall of the viscus and eventually perforates it. ${ }^{6}$ Another possible mechanism was described in a patient with suspected silicone allergy, who developed an inflammatory reaction to the catheter and eventual colon perforation. ${ }^{7}$ The texture of the catheter, age of the patient, and the length of the peritoneal tubing are some of the other factors suspected to play a role in the occurrence of this complication. ${ }^{8}$ With reference to our patient, gradual pressure and irritation by the shunt tube might have caused a fibrous reaction, which, along with necrosis of the bladder wall eventually led to perforation. In some cases, there will be calculus formation which appears to be a foreign-body reaction to the shunt. The management of the bladder perforation must be individualized according to the patient's requirement. Treatment usually comprises the shunt tube removal or exteriorization, treatment of infection, and reinsertion of the shunt tube at an appropriate time. ${ }^{9}$ Retrograde spread of infection resulting in meningitis is usually rare as the distal end of shunt tube will be blocked in most instances. The urine should be sampled for microscopic examination and culture sensitivity. CSF should also be sent for culture and sensitivity. In our case, the encrustation was thin and appeared to be retrieved per urethra. So cystoscopically the portion of the shunt tube seen within the bladder was cut and removed per urethra. The rest of the shunt tube removed through the separate scalp incision. If there is an associated calculus formation, then a cystolithotomy and removal of the calculus along with the cutting of the shunt tube, which is protruding into the bladder, has to be done. Attempts can be made to break the larger calculus around the shunt tube, so that it can be retrieved per urethra. There have also been reports of migration of the distal end of the VP shunt into the stomach, liver, pulmonary artery, inferior vena cava, heart, bowel with or without catheter protrusion from the anus, scrotum, vagina, and transoral protrusion. ${ }^{10,11}$

In our case, the proximal aspect of the shunt demonstrated clear evidence of chronic malfunction without neurological sequelae. The small rent in the bladder caused by shunt tube at its entry gets obliterated once the tube is pulled out per urethra owing to bladder muscle contraction. We also recommend leaving a urethral catheter for 3 weeks for the bladder rent to heal. Although bladder perforation by a ventricular shunt system is rare, it is important for both neurosurgeons and urologists to recognize the potential neurological, urological, and infectious consequences and treat these patients.

\section{Conclusion}

The perforation of the distal end of the VP shunt into the bladder is very rare. A high index of suspicion is needed in diagnosing this problem. This complication requires an operative procedure for correction and affects the quality of life by increasing shunt-related morbidity. Early detection and management of this condition result in a favorable outcome.

\section{References}

1 Agha FP, Amendola MA, Shirazi KK, Amendola BE, Chandler WF. Unusual abdominal complications of ventriculoperitoneal shunts. Radiology 1983;146(2):323-326

2 Grosfeld JL, Cooney DR, Smith J, Campbell RL. Intra-abdominal complications following ventriculoperitoneal shunt procedures. Pediatrics 1974;54(6):791-796

3 Wilson $\mathrm{CB}$, Bertan V. Perforation of the bowel complicating peritoneal shunt for hydrocephalus. Report of two cases. Am Surg 1966;32(9):601-603

4 Kumar R, Sahay S, Gaur B, Singh V. Ascites in ventriculoperitoneal shunt. Indian J Pediatr 2003;70(11):859-864

5 Eichel L, Allende R, Mevorach RA, Hulbert WC, Rabinowitz R. Bladder calculus formation and urinary retention secondary to perforation of a normal bladder by a ventriculoperitoneal shunt. Urology 2002;60(2):344

6 Prasad VS, Krishna AM, Gupta PK. Extrusion of peritoneal catheter of ventriculoperitoneal shunt through the urethra. $\mathrm{Br} J$ Neurosurg 1995;9(2):209-210

7 Brownlee JD, Brodkey JS, Schaefer IK. Colonic perforation by ventriculoperitoneal shunt tubing: a case of suspected silicone allergy. Surg Neurol 1998;49(1):21-24

8 Rahimizadeh A, Haddadina K. Extrusion of a peritoneal catheter. Neurosurgery 1986;18(5):677-678 
9 Ueda Y, Kakino S, Hashimoto O, Imoto K. Perforation of the bladder by a peritoneal catheter: an unusual late complication of ventriculo-peritoneal shunt [in Japanese]. No Shinkei Geka 1998; 26(5):413-416

10 Matsuoka H, Takegami T, Maruyama D, Hamasaki T, Kakita K, Mineura K. Transanal prolapse of a ventriculoperitoneal shunt catheter-case report. Neurol Med Chir (Tokyo) 2008;48(11): 526-528

11 Fewel ME, Garton HJ. Migration of distal ventriculoperitoneal shunt catheter into the heart. Case report and review of the literature. J Neurosurg 2004;100(2, Suppl Pediatrics): 206-211 